\title{
Bases teóricas para el estudio de familias desplazadas
}

\section{Theoretical bases to study displaced families}

\author{
Juan José Flores Flores* \\ Escuela Profesional de Psicología, \\ Universidad de San Martín de Porres, Perú
}

\section{Resumen}

Se presenta una propuesta teórica para el estudio del estado actual en que se encuentran los sistemas familiares desplazados por la violencia política, hoy residentes en Lima. El estudio del tema se justifica, por ser la familia un sistema con responsabilidades en la formación de sus miembros y por constituir el desplazamiento un fenómeno que involucró a miles de personas adultas y menores, con profundo perjuicio en la salud mental de sus miembros y en la estructura económica, social y cultural del país. Para ello, se presenta información sobre el desplazamiento por violencia política, tanto en Colombia como en el Perú, y la propuesta teórica sobre los sistemas familiares para su estudio en el caso de Perú, país en el que se presentó el desplazamiento de miles de familias andinas hacia Lima.

Palabras clave: sistema familiar, desplazamiento, violencia política, desplazado, salud mental.

\begin{abstract}
The article presents a theoretical proposal to study the current state of family systems displaced by political violence, which are now living in Lima. This topic is worth addressing because the family is a system responsible for training its members, and displacement is a phenomenon that has involved
\end{abstract}

Este es un artículo Open Access bajo la licencia Creative Commons Atribución-NoComercial-Compartirlgual 4.0 
thousands of adults and minors causing a profound damage to their mental health, and to the economic, social and cultural structure of the country. To this end, the paper describes displacements due to political violence both in Colombia and Peru, and a theoretical proposal to study family systems in Peru, where the displacement of thousands of Andean families to Lima occurred.

Keywords: family system, displacement, political violence, displaced, mental health.

\section{Introducción}

Este trabajo presenta las bases teóricas para el estudio de las familias desplazadas por la violencia política ocurrida en el interior del país y que hoy residen en Lima.

El tema es abordado por la importancia que tiene, como unidad de análisis, el conocimiento de las familias desplazadas residentes en Lima, en una situación de postviolencia política y dentro de un contexto en que las crisis sociales las afecta. Asimismo, es el producto de la observación y participación que como profesional he desarrollado en la docencia universitaria y en la práctica privada en la ciudad de Ayacucho, durante los años de violencia política, así como, en la responsabilidad al asumir tareas de investigación en el área de desarrollo profesional, y además, en la condición de ciudadano interesado por hacer propuestas frente a los problemas psicosociales propios de nuestra sociedad.

La familia es uno de los sistemas de mayor importancia en el desarrollo de un país, Ferry (como se citó en Leuridan, 2018), al respecto sostiene «que la familia es hoy en día el camino privilegiado para reconstruir una visión política o civilización» ( p. 307) y su estudio genera el interés de diferentes disciplinas como la psicología, la sociología, la antropología, el derecho, entre otras. A la psicología, en particular, le interesa el estudio de los sistemas familiares porque al interior de ellos se establece una estructura cuyos componentes sirven de soporte a un conjunto de interacciones que vivencian sus miembros durante toda su existencia, influyendo en la formación y desarrollo de conductas y procesos psíquicos de los más diversos. 
Trabajar el tema de las familias desplazadas tiene importancia en el Perú; en primer lugar, por ser el desplazamiento un problema a nivel mundial pues «se estimaba que en el mundo había 50 millones de refugiados o desplazados internos, en su mayoría habitantes de países en desarrollo, donde la capacidad para hacer frente a estos problemas era sumamente limitada» (Castellón \& Laplante, 2005, p. 13), y en segundo lugar, por tratarse de un problema latente pues para 1994, según Coral (1994), en el Perú había 120000 familias desplazadas (p. 11). Y es que además de ser desplazadas, desarraigadas de sus lugares de origen, estas familias constituyen una permanente preocupación para el Estado peruano por la exclusión social de la que son víctimas, como lo refiere Coronel (1997):

La condición de desplazado significó la pérdida total de sus derechos: el derecho a la vida, a la integridad personal (recuérdese los múltiples casos de violación de mujeres), a la residencia, a la propiedad, a la protección de la familia, al ejercicio de los derechos políticos. (p. 4)

El desplazamiento provocó crisis paranormativas (González, 2000, p. 2) expresadas en el desmembramiento de las familias ya sea por la pérdida o por la huida de uno de los familiares (recuérdese las cifras de desaparecidos y fallecidos durante el conflicto), con efectos nefastos en la estructura y dinámica familiar de las familias desplazadas.

Estas crisis llevaron a las familias a enfrentar los obstáculos como sucedió con los roles de la mujer y del hombre: ellas comienzan a adaptarse al medio urbano por sus habilidades para las labores domésticas de lavado, cocina, entre otras; ellos por su parte, tienen mayores dificultades pues sus habilidades para el trabajo de campo no le sirven en la ciudad y su inserción laboral fue paulatina haciéndolo preferentemente en las labores que les exigían esfuerzo físico como cargadores, obreros de construcción civil, etc. La población desplazada, al interior de esta crisis, ponía en juego sus formas propias de afrontamiento y de resiliencia para mantenerse como familia.

En este contexto de exclusión social, desfavorable para los sistemas familiares desplazados, consideramos importante establecer algunos hitos teóricos sobre la estructura y la dinámica familiar que pueda servir para el estudio de las familias desplazadas residentes en Lima. Hemos planteado estos 
dos temas del sistema familiar porque «en el análisis del sistema familiar, se pueden destacar, como elementos sincrónicos, la estructura y la dinámica en un momento concreto» (Gobierno de La Rioja, 2003, p. 12).

Un asunto final, y no por ello de menor importancia, es contribuir en todo tiempo a la memoria colectiva de nuestra población sobre un acontecimiento que la afectó gravemente y que, por lo tanto, merece el compromiso social de divulgación científica para prever futuros acontecimientos funestos.

\section{Estado actual del desplazamiento y las familias desplazadas}

\section{Desplazamiento}

Internacional:

El problema del desplazamiento es una de las mayores preocupaciones por la presencia continua de conflictos armados en el mundo; Viancha (2008) señala que, desde la segunda guerra mundial, en el tercer mundo hubo cerca de 22 millones de muertes y más de cien millones de lesionados, lo cual ha dado lugar al desplazamiento hacia otros países (p. 23).

Colombia es uno de los países en América Latina con experiencia similar a Perú en el tema de los desplazados por asuntos de carácter bélico caracterizado por afectar al sistema familiar ya sea por el asesinato de algún miembro de este o por el desplazamiento obligado, tal como se encuentra en el relato siguiente, recogido por Macías, Patemina y Vargas (2004):

Yo vengo desplazada de un pueblito (...), allá llegaron y mataron una hermana mía, mi hijo se voló (...) al otro día que enterramos a mi hermana, yo le dije a mi esposo «vámonos de aquí» (...) mi esposo decía que cómo íbamos a dejar todo ahí, pero yo, quería proteger la vida; agarré y metí lo que pude en una bolsa y nos vinimos para Barranquilla. (p. 99)

Esta declaración confirma lo señalado por Rojas (como se citó en Viancha, 2008), quien sostiene que «las acciones preferidas por los actores armados causantes de los desplazamientos, son: masacres, tomas a poblaciones, 
ataques indiscriminados, combates, amenazas» (p. 27), con el consiguiente abandono de su territorio y sus formas de vida.

Con relación al desplazado en su situación de víctima encontramos una interpretación -desde el enfoque de la resiliencia- de Aristizábal y Palacio (2003), quienes mencionan que en el desplazado hay temores y carencias, pero también capacidad para enfrentar su situación. «Esto implicaría sobrepasar la concepción del desplazado como víctima indefensa del conflicto armado y considerarlo como un sujeto que es responsable de sus actos» (p. 242). Esta postura resiliente es fortalecida en un trabajo de González (2004), quien recoge un testimonio de un miembro de familia desplazada: «La teoría de ellos [Visión Mundial] es muy bonita porque no por el hecho de ser uno desplazado, le van a sentir lástima, no, le dan la oportunidad de que uno trabaje y se gane sus cosas» (p. 11).

Otra vertiente de análisis del tema del desplazamiento por violencia en Colombia, en la que se resalta el componente de personalidad la propone Arango y Cardona (2001): «Este grupo en crisis requiere de un acompañamiento psicosocial en la construcción de nuevos proyectos de vida» (p. 3). Es una propuesta en la que se resalta la importancia de trabajar el componente psicológico con la población desplazada.

\section{Nacional:}

Coronel y Coral son dos autores peruanos que han trabajado el tema del desplazamiento en el Perú; a estas fuentes nos hemos remitido y encontramos coincidencia en sus hallazgos con respecto al número de desplazados, daños psicológicos, trato recibido y el papel de la mujer en el reordenamiento de las familias peruanas desplazadas. Ellas, como parte de la población civil afectada, según Coronel (1997), «en un determinado momento llegaron a ser 600000 personas (156 575 para Ayacucho)» (p. 4); cifras similares a la señalada por Coral (1994): «Para 1994 se consideraba que en el Perú existían 120000 familias que mantienen el estatus de desplazadas» (p. 11). Cohen y Sánchez-Garzoli (2001) afirman que en la década de 1980 «en Perú, una insurgencia virulenta maoísta, junto con la contrainsurgencia gubernamental, desplazaron a aproximadamente 600000 personas» (p. 41), cifra con la cual coincide Aguilar (2007), al señalar 
que en el «Perú se desplazaron aproximadamente 600000 personas, y que para finales de 1990 el número de desplazados disminuyó a 70 000» (p. 8). Coincide con la cifra proporcionada por el Comité de Información del U. S. Committee for Refugees. Cualquiera sea el número de víctimas, las condiciones de vida sociales y psicológicas de los desplazados es preocupante para el científico social, interesado en el desarrollo humano.

El desplazamiento por violencia política fue una nueva forma de migración ya sea desde el interior de Ayacucho hacia la ciudad de Huamanga o de esta hacia Lima, pero bajo condiciones totalmente diferentes a las migraciones tradicionales:

En las zonas más afectadas por la violencia política, la migración tradicional se subsume en el desplazamiento: ambos tienen como factor determinante la violencia política. Esto se refleja en las encuestas que fueron aplicadas entre los que llegaron tanto a Lima como a Huamanga después de 1980, donde no encontramos ningún caso de migración tradicional. (Coral, 1994, p. 8)

Aun en condiciones difíciles, bajo las que se da el desplazamiento, la familia, desde un principio actuó como unidad de integración junto a otras formas de organización, como bien lo apunta Coronel (1997): «En la primera etapa de la violencia política (1980-1985), las formas de organización giran en torno a la familia» (p. 11).

\section{Familia desplazada}

Las familias desplazadas por la violencia política son aquellas que abandonaron sus lugares de origen, exigidas por los conflictos bélicos ocurridos en el Perú a partir de la década de 1980, y se trasladaron a lugares dentro del mismo departamento -desplazamiento intrarregional-o a otros departamentos -desplazados extrarregionales-, Lima principalmente (Coral, 1994, p. 10). Hickel hace una precisión llamándolo explícitamente desplazado interno para diferenciarlo del refugiado: Es «una persona que se ha visto obligada a desplazarse dentro del territorio de su propio país a raíz de un conflicto armado o de tensiones internas» (2001, p. 2). Estas personas, según el mismo Hickel, reciben la protección del derecho internacional. 
Este desplazamiento, violento, sorpresivo, con pérdidas de vida de familiares, abandono de sus tierras con los sembríos quemados al igual que sus animales, tuvo que ocasionar serios trastornos a la vida personal de las víctimas tanto en sus interrelaciones en el hogar como en la propia personalidad, como lo confirma Coronel (1997), «al señalar que la violencia intrafamiliar aumentó por la violencia externa de la que fueron víctimas los miembros del grupo familiar con el perjuicio de su salud mental» (p. 9).

Además de ser desplazados con todo el sufrimiento y perjuicio económico a cuestas, su arribo a otros puntos del país estuvo lleno de dificultades: «En Perú, los campesinos que buscaron acogida en las ciudades, encontraron que el ambiente era ajeno y que no podían hablar español. Por consiguiente, sufrieron aún más de prejuicio y marginación social» (Aguilar, 2007, p. 10).

Incertidumbre, pobreza, prejuicio, marginación, se configuran en el perfil de los miembros de las familias desplazadas. La exclusión social los margina afectando la calidad de vida pues por sus orígenes campesinos no estaban preparados para las labores urbanas lo cual profundiza su pobreza con el consiguiente efecto psicológico sobre los miembros de las familias, tal como lo señala Britton (1998, como se citó en Roca \& Rojas, 2002, p. 704): «La exclusión llega a afectar hasta la autoestima de las personas, lo cual se ve claramente en el caso del desempleo». Democratic catalog, mencionan los efectos diversos de la exclusión: «Es por este motivo que se dice que la exclusión social va más allá del término 'pobreza' y se refiere más bien al sentimiento de marginación» (1995, como se citó en Roca \& Rojas, 2002, p. 704).

El sistema familiar del desplazado fue afectado en su estructura y en su dinámica, por la violencia política desmembrándola en su unidad y afectando las interacciones de sus miembros: «Las familias desplazadas fueron obligadas a abandonar sus hogares o actividades económicas habituales debido a que sus vidas, su seguridad o libertad estaban amenazadas en el contexto de la violencia» (Amérique Latine Histoire et Mémoire, 2005, p. 5). Este abandono de sus lugares de origen bajo situaciones tan dramáticas afecta al sistema familiar en su estructura y a sus miembros en sus dinámicas habituales, como lo informan Castellón y Laplante (2005, p. 14), quienes revisaron un estudio epidemiológico de salud 
mental del Instituto Especializado en Salud Mental H. Delgado-H. Noguchi, en el que se daba cuenta de los problemas de salud mental ocurridos en Lima y en tres regiones de la sierra y en particular Ayacucho, con problemas de depresión, estrés postraumático, alcoholismo y violencia intrafamiliar.

Dentro de este panorama, Forero señala que en Perú, al igual que en Colombia, las familias como sistema, además de ser dañadas tienen que asumir sus responsabilidades como tales, es decir, «aparte de que el destierro ocasiona un fuerte trauma psicológico, el desplazamiento hace recaer casi exclusivamente en ellas la responsabilidad del sostenimiento afectivo y económico de la familia» (2003, p. 7).

\section{Sistema familiar: estructura y dinámica}

Minuchin y Fishman (2004), desde el enfoque sistémico, definen a la familia como un grupo natural, con pautas de interacción que dan lugar a la estructura familiar; es esta estructura familiar -continúan- la que da lugar a las conductas individuales y a la interacción entre ellas, para concluir que «la familia necesita de una estructura viable para desempeñar sus tareas esenciales, a saber, apoyar la individuación al tiempo que proporciona un sentimiento de pertenencia» (p. 25).

Es a partir de esta definición y de la teoría desarrollada por este enfoque, que se propone trabajar el tema de las familias desplazadas.

Las familias son el lugar íntimo de intercambio de subjetividades entre sus miembros lo cual facilita el aprendizaje inicial de una forma de ser persona, formar identidad, desarrollar afectos así como la incorporación de normas y valores. De la Torre (2008), hablando de la identidad como parte del proceso de socialización, sostiene:

Las formas verbales y no verbales de comunicación, los significados y sentidos compartidos, las memorias pasadas, los símbolos, los valores, las actitudes, las tradiciones, los hábitos, las costumbres, los gustos, los prejuicios, hasta las expectativas futuras, son recibidos por los niños y niñas en crecimiento, y por los jóvenes, a través, primero, de los adultos cercanos que cuentan e interpretan las experiencias pasadas. (p. 8) 
En el mismo sentido, referido a los intercambios e interacciones en el proceso de socialización intrafamiliar, encontramos en la Carta del Papa lo siguiente con respecto a la familia: «En su camino familiar, ustedes comparten tantos momentos inolvidables: las comidas, el descanso, las tareas de la casa, la diversión, la oración, las excursiones y peregrinaciones, la solidaridad con los necesitados» (Papa Francisco, 2014, p. 2).

Las conductas señaladas en ambas posturas son formas de interactuar que nos hablan de una dinámica, de una forma de hacer familia. Además, de la construcción intrafamiliar, la familia como un sistema abierto, se relaciona con otros sistemas sociales y está expuesta a recibir todo tipo de influencias y a influenciarlos en una permanente interacción pues como tal, afirma Quintero (2001), es el más representativo de los sistemas humanos, destacando su carácter socioecológico al interior del contexto cultural en el que actúa (p. 4). Baeza (2000) refiere que en la familia se instaura el proceso de socialización del hombre y es en ella donde se dan los lazos afectivos iniciales, la vivencia del tiempo y de las distancias corporales. En ella se hace la historia de la familia a través de las generaciones y es uno de «los pilares de la identidad de una persona» (p. 1).

Con ocasión del XX Aniversario del Año Internacional de la Familia, que se conmemoró en el 2014, la International Federation for Family Development (2014) declaró: «Promote and develop active measures to support the psychological well-being of children and youth with sensitivity to family situations (promover y desarrollar medidas activas que contribuyan al bienestar psicológico de los menores y jóvenes, según la situación de cada familia [en español])» (p. 20), en un documento firmado por 529 representantes de la sociedad civil de 83 países.

\section{Estructura Familiar}

La estructura familiar es uno de los aspectos más ricos para el conocimiento de la familia. Es el espacio en donde se tejen y articulan todas las reglas y roles que regulan su funcionamiento.

Todas las relaciones que establecen los miembros de una familia dan lugar a la conformación de una estructura muy peculiar y propia de cada sistema 
familiar, en el que se advierten subsistemas conformados por las relaciones que establecen los miembros del sistema familiar.

Zevallos (2004) define la estructura familiar a partir de las interacciones relativamente duraderas entre los miembros de la familia, dando lugar a que haya una organización en su interior, en los subsistemas. Se presenta un «invisible conjunto de demandas funcionales que organiza la manera de interactuar entre los miembros de la familia» (p. 42).

Siguiendo a Makabe (2003), convenimos en que la dimensión estructura «se refiere a la composición que organiza el funcionamiento de la familia» (p. 285) resaltando su importancia «porque la trama de las miserias y grandezas de una familia se despliega en el contexto de la estructura familiar, considerándola en sus dimensiones histórica y relacional» (p. 285).

Minuchin y Fishman (2004) señalan que «las pautas de interacción que elabora la familia van a constituir la estructura familiar con la cual sus miembros definen sus conductas y facilitan su interacción recíproca» (p. 25).

Los autores mencionados sostienen el enfoque sistémico para definir la estructura familiar y el concepto de relación e interacción como formadores de conducta de los miembros de la familia. Cibanal (2006) añade la idea de movimiento permanente al decir que: «El concepto de ESTRUCTURA describe la totalidad de las relaciones existentes entre los elementos de un sistema dinámico» (p. 28); estas relaciones van formando, de un lado, un estatus en cada uno de los miembros de dicho sistema y, de otro, establecen los papeles a desempeñar con relación al otro y a todo el conjunto del sistema familiar. El estudio de la estructura de la familia lleva a conocer la dinámica de interacción de sus miembros al interior de ella, lo cual resulta más importante que conocer el número de componentes de la misma, pues nos permite hacer el análisis de las relaciones entre los miembros de los subsistemas y entre estos, tal como también lo sostiene Zuluaga (2004): «Toda familia tiene una organización jerárquica y una estructura de interacción o funcionamiento, y si se modifica la estructura, se producirán cambios en los procesos internos de cada uno de los miembros del sistema» (p. 3). 
Siguiendo el Programa de Trabajo Social y Apoyo a la Dinámica y Estructura Familiar, hemos seleccionado los componentes de la estructura familiar a tener presente en una investigación de campo: subsistemas familiares, vínculos relacionales (resonancia), límites, fronteras y territorialidad (Gobierno de la Rioja, 2003, p. 15).

Subsistemas familiares. Al interior de un sistema familiar se encuentran los subsistemas; estos están conformados por los miembros de la familia que, al interactuar entre ellos, cumplen una serie de funciones con diferentes roles como madre, hijo, esposo, hermano, etc. En un artículo editado por el Gobierno de la Rioja (2003) se lee: «La estructura familiar, la componen los miembros de la familia organizados en subsistemas entre los que existen límites que tienen como objetivo proteger la diferenciación del sistema y facilitar la integración de sus miembros en él» (p. 12).

Los subsistemas ayudan a las familias a cumplir con sus funciones, lo que les permite mantener su estructura. «El cómo se dan las relaciones al interior de los subsistemas y entre estos define a la estructura familiar» (Zevallos, 2004, p. 45).

Diferenciamos los siguientes subsistemas: conyugal, parental y fraternal.

El subsistema conyugal es el conformado por la pareja, responsable inicial de la constitución de la familia, como en su momento lo dijera Aristóteles (2015): «Lo primero que se necesita es unir dos a dos los seres que no pueden ser completos uno sin otro, como el hombre y la mujer para los efectos de la generación» (p. 3). Desde sus inicios está sujeta a un proceso de acomodación con los consiguientes ajustes y desencuentros que se manifiestan en las formas como se relacionan. El vínculo de afecto y la comunidad de intereses constituyen sus características.

El subsistema parental está formado por los padres y los hijos. Este subsistema tiene sus inicios con el nacimiento del primer hijo lo que da lugar a un cambio en las relaciones que hasta ese momento había establecido la pareja. Surgen nuevas exigencias que comprometen a los padres en la medida en que cada cual tiene una idea de lo que le corresponde como responsabilidad de su rol paterno. 
El subsistema fraternal está formado por los hermanos quienes tienen que aprender a convivir en la relación diaria.

Las relaciones que pueden darse entre los subsistemas y al interior de estos, son: alianzas y coaliciones, resonancia, autoridad, áreas de poder, división del trabajo, roles y reglas familiares, vivencia de la sexualidad y flujo de la comunicación.

Vínculos relacionales. Son las relaciones básicas que se establecen entre los miembros de la familia; estos vínculos dan lugar a la resonancia en la proximidad e intensidad emocional en la organización familiar. Esta resonancia es el nivel de sensibilidad que desarrollan los miembros de la familia, y que va desde la cohesión hasta la desunión, dando lugar a que se establezcan grados de diferenciación emocional en la comunicación que ellos establecen.

Límites y fronteras de la familia. Los límites se establecen para separar a los subsistemas; en una familia funcional se espera que los límites sean semipermeables para permitir la individuación y diferenciación necesaria a cada miembro y el apropiado intercambio afectivo entre los integrantes de cada subsistema. Los límites claros permiten una adaptación ideal, armoniosa entre los miembros de los subsistemas y con los otros subsistemas conformantes del gran sistema familiar, los límites difusos significan debilidad o ausencia de ellos y los rígidos marcan una lejanía de relación afectiva, comunicacional. Por otra parte, las fronteras permiten diferenciar a las familias con respecto a otros sistemas propios de la comunidad en la que están establecidas como lo son otras familias e instituciones diversas.

Territorialidad. Cada familia dispone y organiza sus espacios domésticos constituyéndose en lugares íntimos para las transacciones entre sus miembros. La territorialidad es espacial -cocina, sala, etc.- y significante, referida al uso que se da a los espacios -en la sala se reúnen mensualmente, en el dormitorio los padres toman acuerdos sobre los hijos, etc.- (Gobierno de La Rioja, 2003, p. 12). 


\section{Dinámica familiar}

El ser humano vive en permanente compañía con sus semejantes formando grupos de las más diversas características: asociaciones, clubes, amigos, vecinos, partidos políticos, congregaciones, empresas, sindicatos; esta construcción social verificada a través de los años y en las más diversas circunstancias encuentra expresión filosófica en Aristóteles (como se citó en Azcárate, 1873), quien dice que el hombre es un ser social; negarlo y vivir fuera de la sociedad es propio de seres degradados o superiores «y a él pueden aplicarse aquellas palabras de Homero: «Sin familia, sin leyes, sin hogar» (p. 19).

La familia es un sistema abierto y, como lo señalamos, establece relaciones tanto al interior de su propio sistema como con el exterior con otros suprasistemas sociales (escuela, municipalidad, etc.). Al interior de ella son los miembros de los subsistemas los que al establecer relaciones le imprimen una dinámica muy particular y propia a cada familia. Una dinámica llena de fuerza, energía y actividad, coincidiendo con Agudelo al afirmar que «la dinámica familiar comprende las diversas situaciones (2005) de naturaleza psicológica, biológica y social que están presentes en las relaciones que se dan entre los miembros que conforman la familia» (p. 9), lo que les facilita la vida cotidiana llena de comunicación afectiva, autoridad y crianza. Lo señalado por Agudelo (2005), justifica el estudio de la dinámica familiar «para alcanzar el objetivo fundamental de este grupo básico de la sociedad: lograr el crecimiento de los hijos y permitir la continuidad de las familias en una sociedad que está en constante transformación» (p. 9).

En el sistema familiar la dinámica tiene sus propios contenidos formados por «el tejido de relaciones y vínculos atravesados por la colaboración, intercambio, poder y conflicto que se genera entre los miembros de la familia -padre, madre e hijos- al interior de esta» (Gallego, 2012, p. 8).

La dinámica familiar corporizada en las conductas de los miembros del sistema supone el encuentro de subjetividades de emociones, gustos, tendencias, motivaciones, «mediados por una serie de normas, reglas, límites, jerarquías y roles, entre otros, que regulan la convivencia y permite que el funcionamiento de la vida familiar se desarrolle armónicamente» 
(Gallego, 2012, p. 8). Sin embargo, el actuar de cada miembro no siempre es lineal, en concordancia con las normas, y de allí surge otra arista de su importancia: «El estudio de la dinámica familiar surge como una crítica a los supuestos de unidad, interés común y armonía que tradicionalmente se ha dicho acerca de las familias» (Torres, Ortega, Garrido, \& Reyes, 2008, p. 33). Efectivamente, la práctica profesional del psicólogo acumula casuística de familias ganadas por las desavenencias, líos y conflictos que generan interés en el investigador por sistematizar información acerca de ellas.

Categorías de la dinámica familiar. Siguiendo a diversos autores (Agudelo, 2005; Gallego, 2012; Gobierno de la Rioja, 2003), hemos seleccionado las siguientes categorías de la dinámica familiar: asociaciones (alianzas y coaliciones), autoridad y liderazgo, áreas de poder, división del trabajo, roles y reglas familiares, vivencia de la sexualidad y flujo de la comunicación.

La alianza se da al asociarse dos o más miembros de la familia para actuar a favor de ella; mientras que en la coalición, la asociación es para perjudicar a un tercero. Una familia funcional, saludable, establece alianzas basadas en las afinidades y metas conjuntas, evitando las coaliciones que son dañinas a ella. Estas coaliciones o relaciones triangulares pueden ser: triángulo manejado por los padres en conflicto, triángulo por rodeo y triángulo perverso o coalición estable.

Autoridad y liderazgo, caracteriza la jerarquización existente al interior del sistema familiar y se establece en relación con el ciclo vital de la familia, la edad de los padres y de los hijos. Se entiende que un sistema familiar es funcional cuando el subsistema parental asume su autoridad y lidera el hogar al tomar decisiones en acuerdo mutuo, frente al subsistema fraterno y a los suprasistemas.

Áreas de poder, son los espacios domésticos en los que la pareja se relaciona en función a asuntos propios de la dinámica hogareña en la que está implícito el uso del poder para tomar decisiones (adquisición de bienes, destino escolar de los hijos, entre otros). 
División del trabajo, se expresa en la asunción de las tareas diversas dentro del hogar como, por ejemplo, cocinar, lavar, llevar a los niños al centro educativo, ayudar en las tareas escolares. Sara-Laffose (2003) dice que son «los trabajos que realiza la familia en cuanto tal» (p. 184).

Los roles y las reglas familiares se van formando de manera explícita por acuerdo de los miembros de la familia o de manera tácita en la práctica familiar diaria y siempre se irán acuñando por la acumulación de actos repetitivos que se dan en las actividades recíprocas entre los que conforman el hogar. Paulatinamente las conductas se hacen consistentes y cada uno ya sabe qué conducta se espera de él y de los otros. Se manifiestan en las conductas reiteradas que da lugar a actividades recíprocas entre los miembros de la familia, como lo señala Makabe (2003, p. 287).

Vivencia de la sexualidad, es el espacio íntimo de la pareja en el que las responsabilidades, con sus derechos y deberes son acordadas al interior de la díada, de manera tácita o explícita. La relación, además de sus componentes biológicos, tiene una fuerte y decisiva carga psicológica en la vida de sus actores.

Flujo de la comunicación. Para evaluar la calidad, cantidad y dirección de la comunicación en sus vertientes digital (lo que se dice) y analógica (la forma cómo se dice con sus gestos y posturas), en el interior del sistema familiar. Se examinan los patrones formales e informales de la comunicación que establecen los miembros. Cuando la familia es funcional el flujo de comunicación es directo y específico.

Para fortalecer la importancia de la comunicación al interior de las familias, hemos tomado uno de los axiomas de Watzlawick (como se citó en Arango, Rodríguez, Benavides, \& Ubaque, 2016): la imposibilidad de no comunicar «toda interacción trae consigo un mensaje que movilizará la conducta de los participantes» (p. 41).

Estas categorías servirán para el trabajo de información y análisis sobre las características de la dinámica familiar, que junto con la estructura, son las dos dimensiones presentadas para ser trabajadas en el estudio de las familias desplazadas. 
El enfoque teórico presentado puede servir de insumo para el estudio e investigación empírica de la estructura y la dinámica familiar de las familias desplazadas por la violencia política, residentes en Lima, contribuyendo al conocimiento del estado actual en que se encuentran miles de personas y para el establecimiento de políticas públicas de atención en este vasto sector social marginal.

\section{Referencias}

Agudelo, M. E. (2005). Descripción de la dinámica interna de las familias monoparentales, simultáneas, extendidas y compuestas del Municipio de Medellín, vinculadas al proyecto de prevención temprana de la agresión. Revista latinoamericana de ciencias sociales, niñez y juventud, 3(1), 1-19. Recuperado de http:/l www.redalyc.org/pdf/773/77330106.pdf

Aguilar, A. (2007). Marco teórico y aspectos generales relativos al fenómeno de desplazamiento interno forzoso. Recuperado de http://www.eleutheria.ufm.edu/ArticulosPDF/070918_Generales_Relativos_al_fenomeno.pdf

Amérique Latine Histoire et Mémoire. Les Cahiers ALHIM. (2005). Balance del proceso de desplazamiento por violencia política en el Perú (1980-1997). Recuperado de http://alhim.revues.org/647

Arango, M. Z., Rodríguez, A. M., Benavides, M. S., \& Ubaque, S. L. (2016). Los axiomas de la comunicación humana en Paul Watzlawick, Janet Beavin, Don Jackson y su relación con la Terapia Familiar Sistémica. Revista Fundación Universitaria Luis Amigó, 3(1), 33-50. Recuperado de www.funlam.edu.co/revistas/ index.php/RFunlam/article/

Arango, S., \& Cardona, D. (2001). Desplazados: Elementos para su caracterización. Revista de Ciencias Humanas, 8(28), 84-91. Recuperado de ww.utp.edu.co/ chumanas/revistas/revistas/rev28/arango.htm

Aristizábal, E. T., \& Palacio, J. (2003). Subjetivación del acto de desplazarse y aspectos psicopatológicos relacionados con experiencias violentas. Investigación y desarrollo, 11(2), 238-253. Recuperado de http:/l rcientificas.uninorte.edu.co/index.php/investigacion/article/view/1118

Aristóteles. (2015). Política, Libro I. De la sociedad civil. De la esclavitud. De la propiedad. Del poder doméstico. Recuperado de http://biblio.juridicas.unam.mx/libros/2/766/3.pdf

Azcárate, P. (1873). Obras de Aristóteles (tomo 3, pp. 7-21). Madrid. Recuperado de http://www.filosofia.org/cla/ ari/azc03017.htm

Baeza, S. (2000). El rol de la familia en la educación de los hijos. Recuperado de http://iin.oea.org/ Cursos_a_distancia/Lectura10_disc..UT3.pdf

Castellón, R., \& Laplante, L. (2005). Los afectados por el conflicto armado interno del Perú: Exigiendo el derecho a la salud mental. Lima: Ediciones Nova Print. Recuperado de http://www.librosperuanos.com

Cibanal, L. (2006). Introducción a la sistémica y terapia familiar. Recuperado de http://www.editorial-clubuniversitario.es/pdf/575.pdf

Cohen, R., \& Sánchez-Garzoli, G. (2001). El desplazamiento interno en las Américas: Algunas características distintivas. The Brookings-CUNY Project on Internal Displacement. Recuperado de https://www.brookings.edu/ author/gimena-sanchez-garzoli/ 
Coral, I. (1994). Desplazamiento por violencia política en el Perú, 1980-1992. IEP Instituto de Estudios Peruanos. Documento de trabajo $N^{0}$ 58, Serie: Documentos de Política $N^{\circ}$ 6. Perú. Recuperado de www.iep.org.pe. http://archivo.iep.pe/textos/DDT/ddt58.pdf

Coronel, J. (1997). Balance del proceso de desplazamiento por violencia política en el Perú 1980 - 1997. En V. Agreda, A. Diez y M. Glave (Eds.), Perú, el problema agrario en debate (pp. 588-635). Perú: ITDG.

De la Torre, C. (2008). Identidad, identidades y ciencias sociales contemporáneas; conceptos, debates y retos. Psicología Online,1-7. Recuperado de http://www.psicologiaonline.com/articulos/2008/05/identidad_ identidades_y_ciencias_sociales.shtml

Forero, E. (2003). El desplazamiento interno forzado en Colombia. Fundación Ideas para la paz (FIP). Recuperado de http://www.ideaspaz.org/secciones/eventos/download/edgar_forero.pdf

Gallego, A. (2012). Recuperación crítica de los conceptos de familia, dinámica familiar y sus características. Revista Virtual Universidad Católica del Norte, 35(febrero-mayo), 326-345. Recuperado de http:/l revistavirtual.ucn.edu.col

Gobierno de la Rioja. (2003). Programa de trabajo social y apoyo a la dinámica y estructura familiar. Rioja: Logroño. Ochoa Impresores. Recuperado de http://chitita.uta.cl/cursos/2011-1/0000439/recursos/r-12.pdf

González, C. (2004). Transformación y resiliencia en familias desplazadas por la violencia hacia Bogotá. Revista de Estudios Sociales,18(mayo-agosto), 123-130. Recuperado de http://dialnet.unirioja.es/servlet/ articulo?codigo $=2349320$

González, I. (2000). Las crisis familiares. Revista Cubana Medicina General Integral, 16(3). La Habana. Recuperado de http://bvs.sld.cu/revistas/mgi/vol16_3_00/mgi10300.pdf

Hickel, M. (2001). La protección de los desplazados internos afectados por conflictos armados: conceptos y desafíos. Revista Internacional de la Cruz Roja,1-8. Recuperado de https://www.icrc.org/spa/resources/ documents/misc/5tdq4p.htm

International Federation for family Development. (2014). Declaration of the civil society on the occasion of the 20th anniversary of the international year of the family. Recuperado de www.familyperspective.org/decl/ DecBook.pdf

Leuridan, J. (2018). El sentido ético de las dimensiones de la vida ( $2^{\mathrm{a}} \mathrm{Ed}$.). Lima: USMP.

Macías, A., Patemina, A., \& Vargas, K. (2004). Relaciones familiares en familias desplazadas por la violencia ubicadas en «La Cangrejera» (corregimiento de Barranquilla, Colombia). Psicología desde el Caribe. Universidad del Norte, 14, 91-124. Recuperado de http://rcientificas.uninorte.edu.co/index.php/psicologia/ article/viewFile/1781/1161

Makabe, P. (2003). Enfoque sistémico aplicado a la familia. Módulo I: Paradigmas teóricos y marcos de referencia conceptuales. Lima: PUCP.

Minuchin, S., \& Fishman, Ch. (2004). Técnicas de Terapia familiar. Buenos Aires: Paidos.

Papa Francisco (25 de febrero de 2014). Carta del Papa Francisco a las familias del mundo. El Vaticano. Recuperado de https://www.aciprensa.com/noticias/papa-francisco-a-familias-recen-conmigo-para-afrontarlos-retos-con-la-luz-y-la-fuerza-del-evangelio-66077l

Quintero, A. (2001). Escenarios contemporáneos de la familia. Recuperado de www.ts.ucr.ac.cr/binarios/congresos/ reg/slets/slets-017-002.pdf

Roca, I., \& Rojas, B. (2002). Pobreza y exclusión social: una aproximación al caso peruano. Bulletin de l'Institut français d'études andines, 31(3), 699-724. Recuperado de https://journals.openedition.org/bifea/pdf/6828

Sara-Laffose, V. (2003). Familia, cultura y sociedad. Módulo I: Paradigmas teóricos y marcos de referencia conceptuales. Lima: PUCP. 
Torres, L. E., Ortega, P., Garrido, A., \& Reyes, A. G. (2008). Dinámica familiar en familias con hijos e hijas. Revista Intercontinental de Psicología y Educación, 10(2), 31-56. Universidad Intercontinental. México D. F. Recuperado de http://www.redalyc.org/articulo.oa?id=80212387003

Viancha, N. (2008). Representaciones sociales sobre la vida ocupacional de mujeres cabezas de familia desplazadas por la violencia asentadas en altos de Cazuca, Soacha. Recuperado de http:/l bibliotecadigital.usbcali.edu.co/jspui/bitstream/10819/654/1/Representaciones_sociales_sobre_ Viancha_2008.pdf

Zevallos, R. (2004). Enfoques, procesos y modelos de intervención familiar. Módulo II: Modelos y Procesos de intervención familiar, instrumentos técnicos y metodológicos. Lima: PUCP.

Zuluaga, J. (2004). La familia como escenario para la construcción de ciudadanía: una perspectiva desde la socialización en la niñez. Revista latinoamericana de ciencias sociales, niñez y juventud, 2(1). Recuperado de http://www.scielo.org.co/scielo.php?pid=S1692-715X2004000100005\&script=sci_arttext 